\title{
Erratum to: The neurological and ophthalmological manifestations of SPG4-related hereditary spastic paraplegia
}

\author{
Grant Guthrie $^{1,2}$ - Gerald Pfeffer ${ }^{2,3} \cdot$ Maura Bailie $^{1} \cdot$ Karen Bradshaw $^{1,4}$. \\ Andrew C. Browning ${ }^{1}$ Rita Horvath ${ }^{2,3}$ - Patrick F. Chinnery ${ }^{2,3}$. \\ Patrick Yu-Wai-Man ${ }^{1,2,3}$
}

Published online: 2 February 2016

(c) Springer-Verlag Berlin Heidelberg 2016

\section{Erratum to: J Neurol (2013) 260:906-909 DOI 10.1007/s00415-012-6780-3}

There is unfortunately a mistake in the protein notation for one of the SPG4 mutations in Table 1. The c.1253_1255 delAAG mutation was identified in Patient 5. The amino acid change resulting from this deletion is p.Glu418del, not p.E418fsX198.

The correct table is given in the following page.

The online version of the original article can be found under doi:10.1007/s00415-012-6780-3.

Patrick Yu-Wai-Man

Patrick.Yu-Wai-Man@ncl.ac.uk

1 Department of Ophthalmology, Royal Victoria Infirmary, Newcastle upon Tyne, UK

2 Department of Neurology, Royal Victoria Infirmary, Newcastle upon Tyne, UK

3 Wellcome Trust Centre for Mitochondrial Research, Institute of Genetic Medicine,, Newcastle University, Newcastle upon Tyne NE1 3BZ, UK

4 Department of Medical Physics, Royal Victoria Infirmary, Newcastle upon Tyne, UK 
Table 1 Molecular genetic and ophthalmological features of the SPG4 patient cohort

\begin{tabular}{|c|c|c|c|c|c|c|c|c|}
\hline \multirow[t]{2}{*}{ Patient } & \multirow[t]{2}{*}{ Sex } & \multirow{2}{*}{$\begin{array}{l}\text { Age } \\
\text { (years) }\end{array}$} & \multicolumn{2}{|c|}{ SPG4 mutation } & \multirow{2}{*}{$\begin{array}{l}\text { BCVA } \\
\text { RE-LE }\end{array}$} & \multirow{2}{*}{$\begin{array}{l}\text { Optic discs/OCT } \\
\text { measurements }\end{array}$} & \multirow[t]{2}{*}{ Eye movements } & \multirow{2}{*}{$\begin{array}{l}\text { Visual } \\
\text { electrophysiology }\end{array}$} \\
\hline & & & Exon & cDNA change/consequence & & & & \\
\hline 1 & $\mathrm{~F}$ & 31 & 5 & c. $743 \mathrm{C}>\mathrm{G} / \mathrm{p} . \mathrm{S} 245 \mathrm{X}$ & $20 / 20-20 / 20$ & $\begin{array}{l}\text { Normal/no RNFL } \\
\text { thinning }\end{array}$ & Normal & Normal \\
\hline 2 & M & 53 & 5 & c. $743 \mathrm{C}>\mathrm{G} / \mathrm{p} . \mathrm{S} 245 \mathrm{X}$ & $20 / 20-20 / 20$ & $\begin{array}{l}\text { Normal/no RNFL } \\
\text { thinning }\end{array}$ & $\begin{array}{l}\text { Horizontal SWJ/ } \\
\text { saccadic pursuit }\end{array}$ & Normal \\
\hline 3 & $\mathrm{~F}$ & 50 & 6 & c.937delG/p.D313fsX1 & $20 / 20-20 / 20$ & $\begin{array}{l}\text { Normal/no RNFL } \\
\text { thinning }\end{array}$ & Normal & Normal \\
\hline 4 & $\mathrm{~F}$ & 55 & $4-17$ & $\begin{array}{l}\text { del exon 4-17/large-scale } \\
\text { deletion }\end{array}$ & $20 / 20-20 / 20$ & $\begin{array}{l}\text { Normal/no RNFL } \\
\text { thinning }\end{array}$ & Normal & Normal \\
\hline 5 & $\mathrm{~F}$ & 29 & 10 & $\begin{array}{l}\text { c.1253_1255delAAG/ } \\
\text { p.Glu418del }\end{array}$ & $20 / 20-20 / 20$ & $\begin{array}{l}\text { Normal/no RNFL } \\
\text { thinning }\end{array}$ & $\begin{array}{l}\text { Horizontal SWJ/ } \\
\text { saccadic pursuit }\end{array}$ & Normal \\
\hline 6 & $\mathrm{~F}$ & 25 & 11 & c.1442_1443insA/p.V482fsX5 & $20 / 20-20 / 20$ & $\begin{array}{l}\text { Normal/no RNFL } \\
\text { thinning }\end{array}$ & Normal & Normal \\
\hline 7 & $\mathrm{~F}$ & 55 & 11 & c.1442_1443insA/p.V482fsX5 & $20 / 20-20 / 20$ & $\begin{array}{l}\text { Normal/no RNFL } \\
\text { thinning }\end{array}$ & $\begin{array}{l}\text { Horizontal SWJ/ } \\
\text { saccadic pursuit }\end{array}$ & Normal \\
\hline 8 & $\mathrm{~F}$ & 49 & 11 & c. $1414 \mathrm{G}>\mathrm{A} / \mathrm{p} . \mathrm{V} 472 \mathrm{I}$ & $20 / 20-20 / 20$ & $\begin{array}{l}\text { Normal/no RNFL } \\
\text { thinning }\end{array}$ & Normal & Normal \\
\hline 9 & $\mathrm{~F}$ & 72 & 11 & c. $1384 \mathrm{~A}>\mathrm{G} / \mathrm{p} . \mathrm{K} 462 \mathrm{E}$ & $20 / 60-20 / 30$ & $\begin{array}{l}\text { Normal/no RNFL } \\
\text { thinning }\end{array}$ & Normal & Normal \\
\hline 10 & M & 65 & 11 & $\begin{array}{l}\text { c. } 1081 \mathrm{C}>\mathrm{A} ; \mathrm{c} .1082 \mathrm{~T}>\mathrm{A} / \\
\text { p.L361N }\end{array}$ & $20 / 20-20 / 20$ & $\begin{array}{l}\text { Normal/no RNFL } \\
\text { thinning }\end{array}$ & Normal & Normal \\
\hline
\end{tabular}

$B C V A$ best-corrected visual acuities, $c D N A$ complementary DNA, $L E$ left eye, $O C T$ optical coherence tomography, $R E$ right eye, $R N F L$ retinal nerve fibre layer, $S W J$ square wave jerks 